\title{
Review: interactive, multisession, and targeted programmes most effective in preventing eating disorders
}

Stice $\mathrm{E}$, Shaw $\mathrm{H}$. Eating disorder prevention programs: a meta-analytic review. Psychol Bull 2004; 130:206-27.

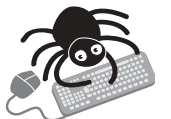

This article

contains extra text on the EBMH website

What are the effects of eating disorder prevention programmes, and which features are associated with the most effective outcomes?

\section{METHODS}

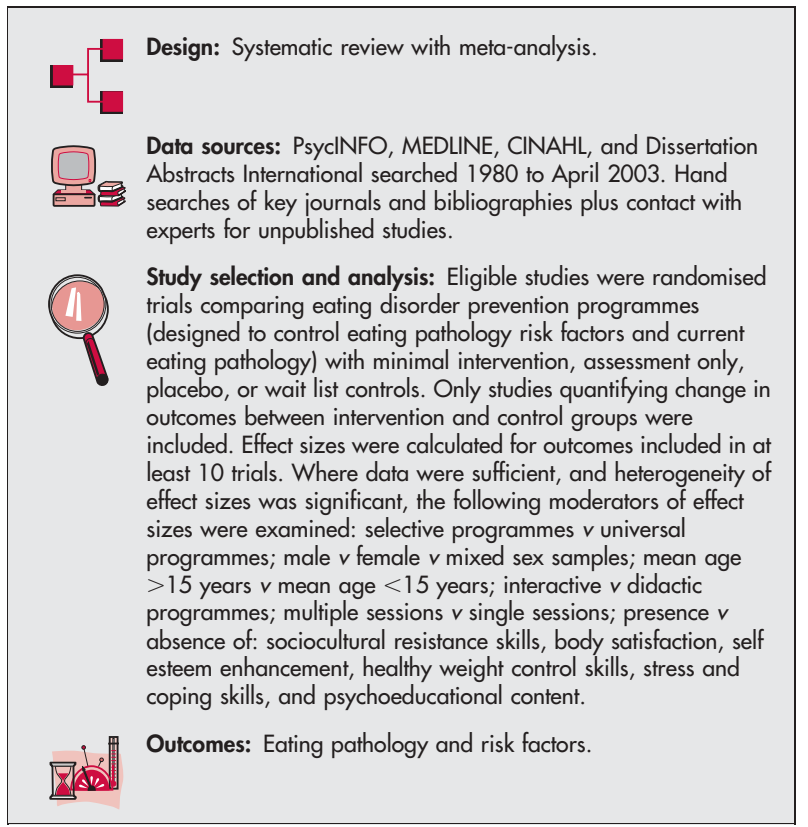

\section{MAIN RESULTS}

Fifty three trials reporting on 38 eating disorder prevention programmes met inclusion criteria. 53\% of the interventions significantly reduced at least one established risk factor. $25 \%$ of the interventions resulted in significant reductions in eating pathology. Mean effect sizes were small to medium (Cohen's criteria) and ranged from 0.11 to 0.38 at trial termination and from 0.05 to 0.29 at follow up (see http://www.ebmentalhealth.com/supplemental for table). In general, larger effect sizes resulted from: selected programmes for high risk individuals compared with unselected samples; interactive versus didactic programmes; participants aged $>15$ years versus younger samples; female only sample versus male samples, and multisession versus single session interventions (see web only table).

For correspondence: Eric Stice, Department of Psychology, University of Texas, Austin, Texas, USA, 78712; stice@psy.utexas.edu

Sources of funding: This study was funded by the National Institutes of Health.

\section{CONCLUSIONS}

Success has been shown with some types of eating disorder prevention programmes. The largest benefits were found with programmes that were interactive, multisession, and targeted, particularly for females and those over age 15 .

\section{Commentary}

B odyweight, and shape concerns, and dieting and exercise disorders represent common sources of distress in females, with subclinical disorders affecting $10 \%-25 \%$ of women and eating disorders affecting up to $5 \%$. ${ }^{1}$ The development of programmes for preventing eating disorders, or associated risk factor, or both have arisen from concerns about the existing and increasing prevalence of such disorders.

Two previous reviews ${ }^{12}$ were discouraging about the potential for preventing eating disorders. Austin's review concluded that only four of 20 interventions showed any positive behaviour change; seven found improvements in attitudes or concerns about weight and shape; whereas four showed worsening of symptoms post-intervention. ${ }^{1}$ In contrast, Pratt and Woolfenden included 13 trials, and found insufficient evidence to conclude prevention or harm from any prevention programme. ${ }^{2}$

This review by Stice and Shaw used broader inclusion criteria and more recent studies. It is encouraging to note that small to moderate effect sizes were found. The studies are most frequently from the US, but also include those from Canada, Australia, Israel, UK, Switzerland, and Italy, making the results geographically generalisable; yet evidence of impact with ethnic diversity has not yet been adequately assessed.

In addition to providing an update, the value of this review is in the analyses of the factors associated with larger effects. Although the direct tests of the moderator variables identified in the meta-analyses will provide areas for further research, there are many encouraging findings with implications for clinical and community work. The analyses lend support to interventions with high risk individuals, particularly with targeted programmes for females over 15 years of age. Cognitive and behavioural interventions should be pursued in both clinical and school based interventions. As expected, multiple sessions were found to be more effective than single, yet those in the range of 4-5 sessions showed a positive impact. This means that the less targeted, more population based interventions to girls in schools have the potential to change attitudes and behaviours without making the interventions so intensive they would not fit into the time or resources of the school system. This review has significantly added to our knowledge regarding eating disorder preventive interventions.

Donna Ciliska, RN, PhD McMaster University, Hamilton, Ontario, Canada

1 Austin SB. Prevention research in eating disorders: theory and new directions. Psych Med 2000;30:1249-62.

2 Pratt BM, Woolfenden SR. Interventions for preventing eating disorders in children and adolescents (Cochrane Review). In: The Cochrane Library. Oxford: Update Software, 2003:3. 